\title{
Equipment for linking the AutoAnalyzer on-line to a computer
}

\author{
D. SIMPSON, G. E. SIMS, M. I. HARRISON, AND L. G. WHITBY
}

From the Department of Clinical Chemistry, The Royal Infirmary of Edinburgh, and Elliott Medical Automation $\vec{\circ}$ Ltd, Borehamwood, Herts

SYNOPSIS An Elliott 903 computer with $8 \mathrm{~K}$ central core store and magnetic tape backing store has i been operated for approximately 20 months in a clinical chemistry laboratory. Details of the equip- $\dot{\sim}$ ment designed for linking AutoAnalyzers on-line to the computer are described, and data presented $\overrightarrow{\vec{V}}$ concerning the time required by the computer for different processes. The reliability of the various components in daily operation is discussed.

Limitations in the system's capabilities have been defined, and ways of overcoming these are delineated. At present, routine operations include the preparation of worksheets for a limited range of tests (five channels), monitoring of up to 11 AutoAnalyzer channels at a time on a seven-day $\stackrel{\Im}{工}$ week basis (with process control and automatic calculation of results), and the provision of quality control data. Cumulative reports can be printed out on those analyses for which computer-prepared . worksheets are provided but the system will require extension before these can be issued sufficiently rapidly for routine use.

Flynn, Piper, and Roberts (1966) described a system for peak recognition and analogue to digital conversion that enabled the raw output from five AutoAnalyzer ${ }^{1}$ channels to be collected in a form suitable for processing by an off-line computer. Experience with the system in this laboratory drew attention to the disadvantages of off-line batch processing by a computer of the output from automatic analysers (Whitby, Proffitt, and McMaster, 1968), and a dedicated computer system was later obtained to meet this laboratory's principal requirements. The chemical validation of this latter system has been reported (Whitby and Simpson, 1969), and this paper describes the performance of the system in daily operation. Technical features of the special hardware, developed with support from the National Research Development Corporation, are detailed; a preliminary account of the special hardware has been published (Griffiths and Carter, 1969).

\section{Description of the System}

The central processor is a standard Elliott ${ }^{2}$ 903C general purpose digital computer with an 8192

Received for publication 30 July 1970.

${ }^{1}$ Technicon Instruments Co Ltd, Hanworth Lane, Chertsey, Surrey. ${ }^{2}$ Elliott Medical Automation Ltd, Elstree Way, Borehamwood, Herts. 18-bit word store, with a backing store of three industry-compatible Ampex TM7 tape handlers $(9 \mathrm{kch} / \mathrm{sec})$ linked to the central processor via a tape controller and multiplexer control.

The standard input devices consist of an on-line Teletype and a paper tape reader $(250 \mathrm{ch} / \mathrm{sec} ; 8$-hole, ISO code). The central processor also receives:signals on-line from AutoAnalyzers, these signals being amplified by analyser consoles situated on the $\dot{\delta}$ laboratory bench and transmitted to the dataacquisition unit which is about $20 \mathrm{~m}$ away in the 0 computer room; these units of special hardware, and features of their operation, will be described later.

Output from the computer is in several forms. $\stackrel{\sim}{\sigma}$ High priority messages (eg, error messages requiring $N$ a decision by the operator, emergency results) are printed up on the on-line Teletype keyboard. Lower priority messages (eg, worksheets or statistical calculations) are issued via the paper tape punch for $\frac{0}{\Phi}$ printing up off-line. Reports are output initially on $\stackrel{\Phi}{\mathcal{S}}$ paper tape and are then either read in again for 7 printing up on-line on an IBM $(15 \mathrm{ch} / \mathrm{sec})$ printer or off-line on a model 35 Teletype $(10 \mathrm{ch} / \mathrm{sec})$.

The computer has four priority interrupt levels for $\frac{P}{D}$ programming purposes. Level 1 is used for entering $\frac{2}{\circ}$ programs and for testing the system. When operat- 
ing, the system is normally functioning at the base level, level 4. Every two seconds, however, when on-line to AutoAnalyzers, the base level program is halted temporarily and control passed to level 2, when the on-line channels are read into the computer and validated peaks stored; level 2 programs are held in the core store. Normally control then returns to level 4 , where the original program is continued, but if a message is waiting to be input on the Teletype this takes priority on level 3 . The programs which are run on level 4 are either held in the core store or on magnetic tape, and transferred to the core store when required. Programs to be run on level 4 are arranged in order of urgency in a level 4 queue, the output of error messages being followed in order of priority by calculation of concentrations, setting up procedures for analytical channels, output of emergency results and quality control messages, input of patient and specimen data, preparation of worksheets, and output of results, etc. The change from one level of priority to another is achieved by a combination of hardware and software. The allocation of programs to the various priority levels, and their order of priority within these levels, differs in several ways from the system described by Griffiths and Carter (1969) in which there was no magnetic tape backing store.

The programming language used in the system is 903 symbolic input routine (SIR). This is an assembly language which has a one to one correspondence with machine code, so is efficient in computer space and time. It is more powerful than machine code, eg, locations can be referred to by names instead of numbers, constants can be referred to directly in the address part of an instruction, and programs can be divided into variable length blocks or modules, simplifying integration of routines written by different people.

\section{SPECIAL HARDWARE}

Analyser consoles

These tap signals separately from the reference and measuring photocells of the detector units and the voltages are amplified a thousand times if the maximum output is $+10 \mathrm{mV}$, or ten thousand times if the maximum output is $+1 \mathrm{mV}$; the amplification unit has very low drift characteristics. The consoles also attenuate these amplified outputs to levels within the range $0-20 \mathrm{mV}$, for transmission to standard AutoAnalyzer strip-chart recorders required for back-up purposes and for the full interpretation of error messages issued by the computer. With the exception of the first, each console can be provided with eight amplifier boards and be connected to two groups of analysers made up of various channel combinations, eg, five in group $\mathbf{A}$ and three in group B. The first console has one channel used for internal reference and standard voltage measurements.

The controls on the analyser console allow the individual output levels of the reference and measuring photocells on-line to the console to be displayed in turn on the meter, or the performance of the corresponding amplifier boards to be inspected and adjusted when equipment is being set up. On-line monitoring of analysers is then initiated with the 'on-line switch', and a green light indicates that a set of channels is on-line. If a fault is detected, the red light associated with that switch glows for one minute and an explanatory message is issued by the computer; the operation of these lights is duplicated on the control panel of the data acquisition unit.

\section{Data acquisition unit}

The data acquisition unit receives the 0 to $+10 \mathrm{~V}$ analogue DC voltages from the analyser consoles. It includes a multiplexer and multiplexer controller, analogue selection unit, timer, status word and fault registers, and analogue-to-digital converter, and it converts these voltages to their binary equivalents. Up to 11 channels (22 analogue inputs) of AutoAnalyzer equipment have been operated simultaneously on-line (Whitby and Simpson, 1969).

The multiplexer in the data acquisition unit expands the computer's single standard peripheral interface into two such interfaces and into a special interface for the connexion of the on-line printer. Input to and output from the computer is decoded by the multiplexer and controller and transmitted to the analogue selection unit, which selects the required channel and connects it to the analogue-todigital converter.

The reference and measuring subchannels from the first analyser console are used for checking purposes. The reference subchannel is connected to a precision voltage reference source to monitor the accuracy of the analogue-to-digital converter, and the input to the measuring subchannel is disconnected so that any switch leakage, noise, or drift in the analogue-to-digital converter may be detected.

A status word register, consisting of an 18-bit gate board, monitors the switches on the analyser consoles, timer, and paper tape equipment, and passes this information to the multiplexer controller. The timer unit outputs a pulse every $2 \mathrm{~s}$; this is used as a level 2 interrupt to hold up the program currently being processed for about $15 \mathrm{~ms}$. During this time, the computer reads the measuring and reference signals from the photocells that are on-line before returning to its previous level of operation. As each channel is read, the 0 to $+10 \mathrm{~V}$ analogue signal is inverted and halved in the analogue-to- 


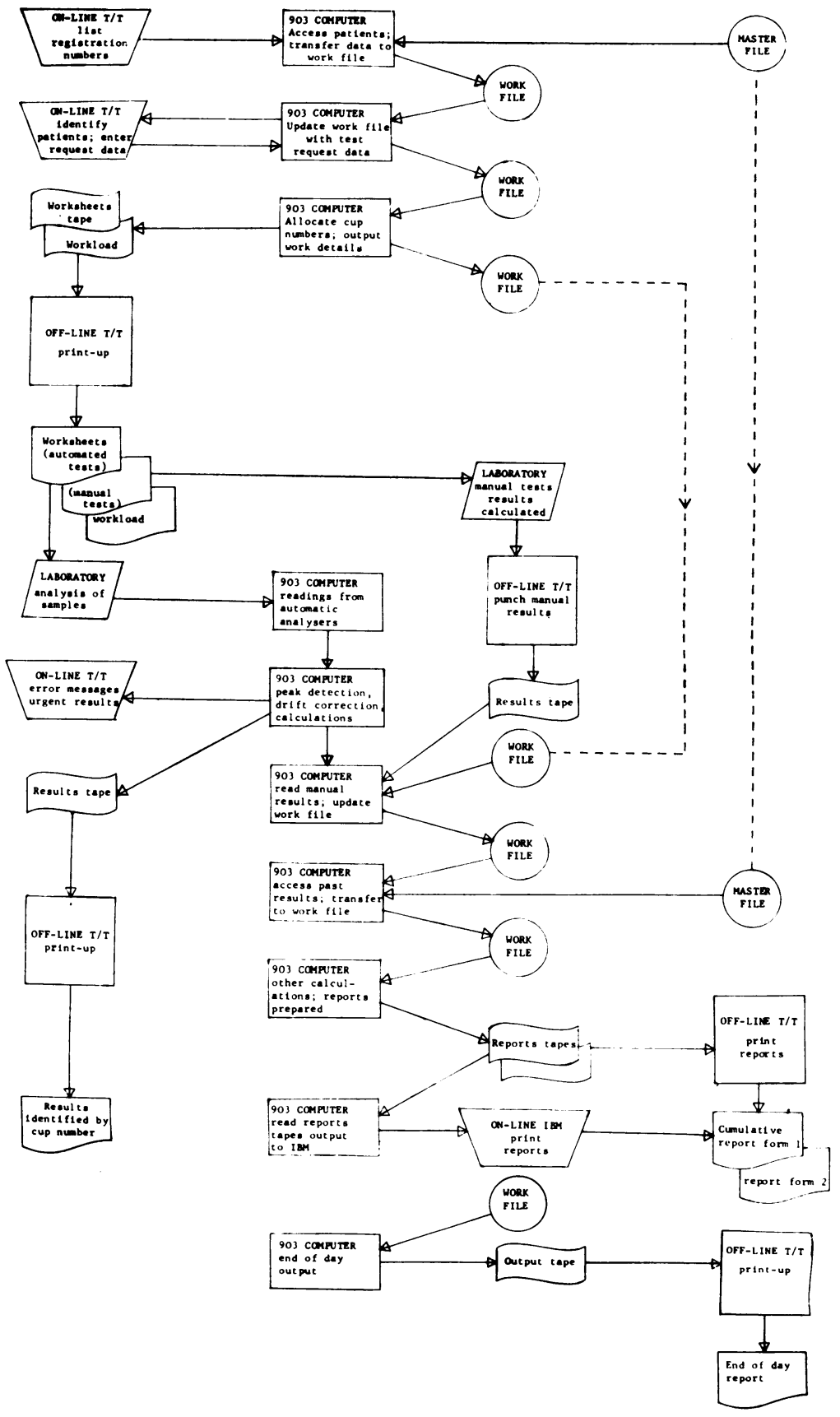

Fig. 1. Simplified flow diagram of the various operations carried out with an Elliott 903 computer working in a clinical chemistry laboratory. 
digital converter amplifier, and the 0 to $-5 \mathrm{~V}$ output is converted in about $100 \mu \mathrm{s}$ into a 12-bit digital representation by the analogue-to-digital converter. After this representation has been examined by the software, the computer addresses the fault register which activates the indicator lamps. With the computer on-line to analytical equipment, if the processor were to address a faulty peripheral and receive no reply, the whole system could remain idle for a considerable time before the fault was noticed; to prevent this, a pseudo-reply circuit limits the waiting time to $300 \mu \mathrm{s}$, after which a message is printed out by the software on the on-line Teletype.

\section{Software and Operational Procedures}

The on-line system programs are located on the first few blocks of each magnetic tape file. When a program is required a search routine selects one of the handlers, reads the program, and places it in the computer core store. The required program may be called by a previous program through the level 4 queue, as in the calculation of concentrations, or it may be called from the on-line Teletype by input of the appropriate code.

The master file tape consists of three main sections: programs, patient identification records, and patient results records. The work file tapes have in addition test request data for all specimens with work still to be completed. Master and work file data are arranged in registration number order in variable length blocks (maximum 512 words) containing records also of variable length.

At the start of a day's operation, a trigger program is input through the reader to bring into store the system program, which includes the level 2 and 3 programs and level 4 queue processing program as well as peak detection parameters, channel allocations, standards concentrations, etc: it also opens up the magnetic tape files. Throughout the day the work file is updated by writing from one work file tape to the other, adding the latest request data entered on the Teletype. At the end of the day's run, request and results data relating to completed work are transferred to the master file and a new master file is prepared. A simplified flow diagram of the overall system is shown in Fig. 1 and the individual procedures are explained in greater detail in the following sections.

\section{SETTING-UP PROCEDURES}

The program used for input of the date, number of first specimen received on this date, changes in AutoAnalyzer group and channel allocations, etc, is called down from magnetic tape by typing in a message. Channels may be re-allocated via the on- line Teletype at any time during the day provided that, at the time of re-allocation, the channel is not on-line to the computer.

Commercial control sera are used for preparing calibration and drift control standards for certain tests, eg, for total protein and for bilirubin. Since the composition of control sera varies from batch to batch, the facility to change the concentration of standards at the start of an analytical run is essential; this is effected by altering the value stored in the appropriate location. Other pooled sera are used for quality control purposes, and the computer-calculated concentrations are checked against predetermined values throughout an analytical run; the mean and SD for the constituents of the serum are entered at the start.

INPUT OF PATIENT AND TEST REQUEST DATA This can occur via the on-line Teletype in two stages using a conversational mode (Whitby and Simpson, 1969). These data are stored until a block of patient and test request data has been entered when they are written to the magnetic tape files. The conversational mode of input proved too slow for routine use, partly because of shortcomings in the method of identifying patients, and an alternative off-line method of input is now being developed.

\section{OUTPUT OF WORKLOAD AND WORKSHEETS}

Statements of the outstanding workload are output to the paper tape punch when requested via the on-line Teletype, and worksheets printed up on the basis of this information. Calibration standards occupy positions $1-8$, drift standards positions 9,10 , 20,30 , etc, and pooled serum positions $19,29,39$, etc. Samples from patients are allocated to worksheets automatically in a priority order: emergency requests, followed by specimens requiring their analysis repeated because of a fault on first analysis, then samples held over from the previous day, and finally the day's samples in order of accession number. A 'change cup number' routine can be used for very urgent specimens. Worksheets can be provided for analyses carried out off-line as well as for on-line work.

\section{DATA ACQUISITION AND PEAK DETECTION}

The program reads the output of the reference and measuring photocells, checks that the reference value is within prescribed limits of its original setting, divides the measuring cell reading by the reference cell reading, and stores the result for that channel. When a series of readings for a particular channel has been stored, the program enters a peak detection routine. The basic program for peak detection and validation is applicable to all channels but 
its action on any particular channel is determined by a series of parameters evaluated for each particular test (Whitby and Simpson, 1969); these are transferred to core at the start of a run. Parameters can be changed by entering the setting-up procedures routine, typing the address of the appropriate location, and changing the value stored in this location.

If a peak fails to conform to the peak validation requirements, an error message is output and the fault lights show for that channel until the next acceptable peak is detected. If four consecutive faulty peaks are detected (eg, due to a block in the sample line), a special message is output but the computer can be instructed to continue its search for peaks while the block is cleared-provided the sampler plate is not stopped, the timing sequence is maintained and if satisfactory peaks are once more obtained they can still be accepted by the computer program.

\section{STANDARDS IDENTIFICATION AND}

VALIDATION

The maximum number of calibration standards is eight. Identification is based on a search procedure which examines the difference in transmission units between consecutive peaks and compares them with a predetermined pattern of differences (Whitby and Simpson, 1969).

\section{CALCULATION OF CONCENTRATIONS}

The program calculates the difference between successive drift standards, and compares each with the first drift standard for a run. As long as drift is within preset limits, the program then applies a linear correction for drift to the nine specimens analysed between adjacent drift standards. If a drift standard falls outside allowable limits, a message is output, and the operator must indicate whether to use, restart, or cancel; if the use option is selected, this suppresses the faulty drift standard and drift is calculated over a double interval. The drift-corrected peak heights are interpolated against the calibration standards and the concentration of the samples is determined.

\section{OUTPUT OF RESULTS}

Emergency tests, and results for a control serum (position 19, 29, etc) falling outside prescribed limits, are output on the on-line Teletype as soon as they become available. Other results are output in groups of eight via the tape punch identified by cup number, and are available for back-up reporting purposes in the event of subsequent system failure (when they could be printed up off-line); they are also written on to the work file and associated with the appropriate records.
Cumulative reports are output via the tape punch in batches of 45 in response to a message on the online Teletype; these reports can only be provided for $\stackrel{\vec{S}}{\stackrel{P}{*}}$ those analyses that have computer-prepared work- $\bar{O}$ sheets. Additional calculations (eg, anion-cation differences, creatinine clearances) are carried out by the program after receiving an 'output of reports' instruction. The tape bearing the reports can be read in again for printing up on the on-line printer on a time-sharing basis or printed up off-line on preprinted continuous stationery (Whitby and Simpson, 1969).

QUALITY CONTROL

Real time quality control depends on the results ? obtained for the specimens of pooled serum placed $\vec{V}$ in positions 19, 29, etc.; these are compared with the mean and SD values input at the start of the analytical run. If the calculated and stated values differ by more than $2 \mathrm{SD}$, or if two consecutive results differ from the stated value by more than $1.5 \mathrm{SD}$ in the same direction, or if three successive values by more than $1 \mathrm{SD}$ in the same direction, a warning message is output and the value for the current pool printed together with the two preceding pool values. Other quality control specimens are analysed during each analytical run as if they were samples from patients, and values suitable for cusum plots are available at the end of each batch of determinations.

END-OF-DAY OUTPUT

A summary is output to the paper tape punch as part of a closedown procedure. This gives statistical information, a statement on workload, a list of all requests entered that day, and results for completed analyses and certain additional calculations, eg, creatinine clearances. The data are output in registration number order and can be readily consulted when answering enquiries.

\section{FILE UPDATING PROCEDURES}

A paper tape program for updating files is input after $\mathcal{N}$ the closedown procedures. In the first part of this $N$ routine, all programs and cumulative records are read from the master file and updated by adding records of completed work from the work file to produce a new master file. In the second part, all incomplete tests are read from the work file to $\stackrel{D}{\rightarrow}$ produce a new work file. At the end of the updating 7 routine, the program outputs to the Teletype the number of blocks of information stored on the $\overrightarrow{\mathbb{D}}$ master file and an estimate of how long the file can $\frac{O}{\mathbb{Q}}$ be used before having to remove patients to a $\frac{\varrho}{\sigma}$ history file. 


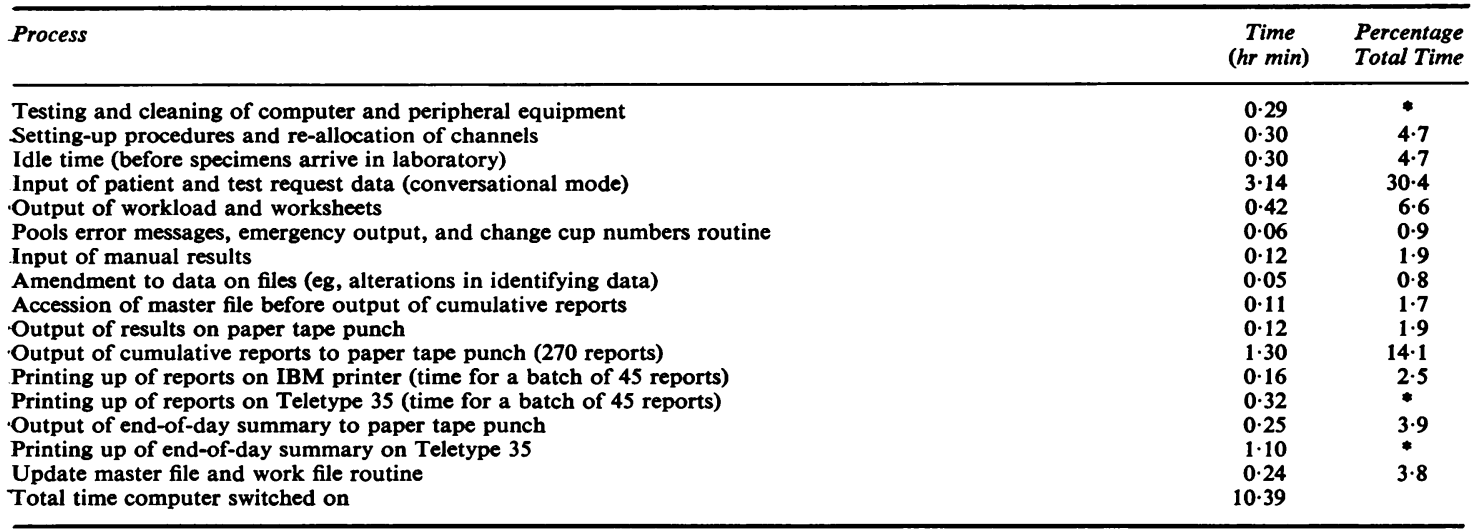

Table I Analysis of one day's operations performed by the computer ${ }^{1}$

${ }^{1}$ Activities indicated with'an asterisk represent off-line activities. The column headed 'Percentage Total Time' does not add up to $100 \%$, since it does not include timings for the routine carried out when searching for and checking standard curves, nor for peak evaluation and output of peak detection and other error messages on the on-line Teletype, nor for updating of magnetic tape work files with test results. The detailed assessment of time spent in on-line monitoring of various aspects of AutoAnalyzer operation would also be difficult to assess.

\section{Operation of the System}

The validation of several individual aspects of the system, particularly the chemical validation, has already been described (Whitby and Simpson, 1969) and the data presented here are mainly concerned with the operation of the integrated system. Although the computer has contributed greatly to the operation of the laboratory, some procedures are carried out too slowly with the present hardware configuration for them to be taken over by the computer as routine operations. The two most timeconsuming procedures in on-line operation on an average working day are the input of patient data by the conversational mode and the output of cumulative reports. The third bottleneck, transfer of data to and from magnetic tape files, is closely associated with the input and output timing difficulties.

Table I sets out an analysis of one day's operations. The computer is switched on at 08.00 and switched off at approximately 18.30. The data presented in this table were collected at a time when there were approximately 3,000 patients on the master file. At the start of the day's operations there were 30 patients on the work file, and at the end of the day's analytical work there were 310 records on the work file before updating the master file.

The time taken to enter data for 24 patients when the computer is monitoring six to nine AutoAnalyzer channels is 21 minutes (range 17-35 minutes), and similarly the time to output a batch of 45 cumulative reports to the tape punch is 13 minutes (range 12-16 minutes). The time for printing up 45 reports on the on-line printer when the computer is monitoring up to 11 AutoAnalyzer channels is 16 minutes; this reduces slightly, to 15 minutes, when the computer is not doing other work. The slowness of these input and output operations means that the system cannot be used routinely for the issue of cumulative reports; non-cumulative reports using combined request and report forms (Whitby et al, 1968) are still being prepared by hand, which means a lot of transcription.

The reliability of the various components has been assessed over a period of 20 months (Table II). A percentage down time for the computer of $0.4 \%$ would appear to be an acceptable value, but the peripheral down time of $6.6 \%$ is high; this reduces to $4 \cdot 1 \%$ if the effect of an initial design deficiency on the magnetic tape controller is discounted. All the peripherals have developed faults; the frequency and duration of breakdown for the different components has varied considerably. The length of each breakdown has been closely related to the ease with which the fault could be detected, but the time to secure the services of an engineer and the availability of replacement components have also been important features.

\section{Discussion}

The on-line system described in this paper has several advantages compared with the off-line system which used an Elliott 803 computer in a batchprocessing mode (Flynn et al, 1966; Whitby et al, 1968). It incorporates process control features which permit real time detection of faults in the operation of the analytical equipment, starting with the valida- 


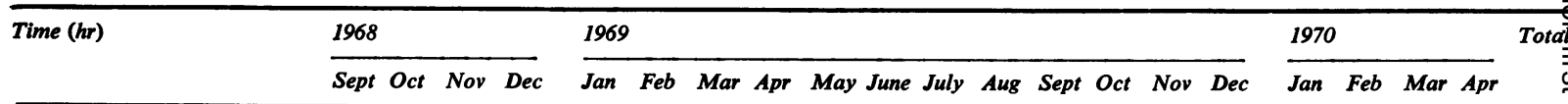

Total Time

Total time computer switched on

Total time computer in use

Total computer down time \begin{tabular}{rrrr}
166 & 145 & 172 & 182 \\
\hline & - & - & -
\end{tabular} $\begin{array}{llll}168 & 208 & 237 & 190\end{array}$ $\begin{array}{rrrrrrrrrrrrr}189 & 198 & 203 & 232 & 256 & 239 & 248 & 195 & 213 & 256 & 228 & 241 \\ - & - & 1 & - & 8 & - & - & 9 & - & - & & -\end{array}$ $\begin{array}{llllllllllll}196 & 213 & 216 & 248 & 269 & 239 & 250 & 227 & 214 & 259 & 229 & 242\end{array}$

$\begin{array}{lllll}230 & 241 & 266 & 250 & 4,592 \mathrm{C}\end{array}$

$211220226 \quad 212$ 4,2725(93.

Peripheral Down Time

DAU

Reader

Punch

Teletype

Magnetic tape controller

Magnetic tape handler

Total peripheral down time

Servicing

Routine servicing by engineer

Out-of-hours servicing by

engineer

Modifications to hardware

Cleaning and testing by

operator

$$
\begin{array}{lrrr}
- & 6 & - & - \\
2 & 11 & 9 & - \\
- & - & - & 1 \\
- & 46 & 56 & - \\
\overline{2} & \overline{63} & \overline{65} & 8
\end{array}
$$

$$
\begin{array}{ll}
12 & 16 \\
- & - \\
- & - \\
- & -
\end{array}
$$

$$
\begin{aligned}
& -\quad \\
& -\quad \\
& -\quad \\
& 5= \\
& 5-
\end{aligned}
$$

$$
\begin{array}{rr}
- & 10 \\
- & - \\
- & \frac{10}{2} \\
2 & 3 \\
2 & 23
\end{array}
$$$$
\begin{array}{rrrr}
- & - & - & - \\
- & 2 & - & - \\
- & - & - & - \\
1 & - & - & - \\
1 & 3 & 1 & 1
\end{array}
$$$$
\begin{aligned}
& -4-- \\
& -\quad- \\
& -\quad- \\
& \overline{1}-\overline{-} \\
& 19 \\
& 19 \\
& 21
\end{aligned}
$$

$$
\begin{array}{rrrrrrrrrrrrrrrrrrrrrrrrrrr}
- & - & - & - & - & - & - & - & - & 9 & & 13 & - & 18 & - & 17 & - & 10 & - & 9 \\
- & 12 & 51 & - & - & 6 & - & - & 11 & 3 & - & 4 & - & 2 & - & 3 & - & -3 & - & 12 \\
- & - & 8 & - & - & - & 17 & - & - & - & - & - & - & - & - & - & - & - & - & - \\
6 & 8 & 6 & 15 & 16 & 21 & 22 & 19 & 12 & 17 & 12 & 10 & 7 & 11 & 6 & 3 & 2 & 3 & 2 & 2
\end{array}
$$

Table II Summary of operation of system for 20-month period

tion of calibration curves and continuing with the assessment of individual peaks, the measurement of drift, and the monitoring of concentrations observed when a control serum is analysed repeatedly; with the off-line system none of these assessments could be performed automatically until the end of an analytical run and only some could be included in the off-line programs. With the on-line system, also, when analyses are urgently requested, not only can the specimen be automatically advanced in the queue, but the results are printed out on-line for immediate reporting; with the off-line system, interchange of specimens could be performed (with the risk of confusing their identity) but urgent calculations had to be performed without the computer. Fault-recognition procedures have worked well with the on-line system, and simple restart procedures can be performed, unlike with the off-line system where it was necessary to begin with a new set of calibration standards. Whenever the on-line system rejects a peak (eg, faulty, too high a concentration, etc), the specimen is automatically allocated a place with high priority on the next worksheet; these reallocation procedures could not be carried out automatically with the off-line system.

Some components of the present system operate too slowly, and the computer's routine tasks are at present restricted to the preparation of worksheets for a limited range of laboratory tests (five channels), the monitoring of up to 11 AutoAnalyzer channels at a time on a seven-day week basis (with process control and automatic calculation of results), and the provision of quality control data. On the input side, the conversational mode of entering data from request forms via the on-line Teletype proved to be slower than had been anticipated; this is being replaced by an off-line batch method of entry, which should later allow the use of request forms bearing patient identification data in machine-readable form. On the output side, the equipment prints up cumulative reports too slowly, but delays are also due to the limited space available in the central processor for drawing up each cumulative report before output. The present intention is to enlarge the core store to $16 \mathrm{~K}$ and to attach a line printer to overcome these hardware limitations.

This project has been generously supported by a grant from the South-Eastern Regional Hospital Board, Scotland, and has been carried out under the auspices of the National Research Development Corporation. We wish to thank the many colleagues and members of staff who have helped with the conduct of the work.

\section{References}

Flynn, F. V., Piper, K. A., and Roberts, P. K. (1966). Equipment for linking the AutoAnalyzer to an off-line computer. J. clin. Path., 19, 633-639.

Griffiths, P. D., and Carter, N. W. (1969). On-line acquisition of the output of AutoAnalyzers. J. clin. Path., 22, 609-616.

Whitby, L. G., Proffitt, J., and McMaster, R. S. (1968). Experience with off-line processing by computer of chemical laboratory data. Scot. med. J., 13, 181-191.

Whitby, L. G., and Simpson, D. (1969). Experience with on-line computing in clinical chemistry. J. clin. Path., 22, Suppl. (Coll. Path.), 3, 107-124. 